\title{
QPSK Phase and Amplitude Regeneration at 56 Gbaud in a Novel Idler-Free Non-Degenerate Phase Sensitive Amplifier
}

\author{
Joseph Kakande $^{1 *}$, Adonis Bogris ${ }^{2}$, Radan Slavík $^{1}$, Francesca Parmigiani ${ }^{1}$, \\ Dimitris Syvridis ${ }^{3}$, Mats Sköld ${ }^{4}$, Mathias Westlund ${ }^{4}$, Periklis Petropoulos ${ }^{1}$, and David J. Richardson ${ }^{1}$ \\ (1) Optoelectronics Research Centre, University of Southampton, Southampton, SO17 1BJ, UK \\ (2) Department of Informatics, Technological Educational Institute of Athens, Aghiou Spiridonos, 12210 Egaleo, Athens, Greece \\ (3) National and Kapodistrian University of Athens, Panepistimiopolis, Ilissia, 15784, Athens, Greece \\ (4) EXFO Sweden AB, Arvid Hedvalls backe 4, SE-41133, Gothenburg, Sweden \\ *jkk@orc.soton.ac.uk
}

\begin{abstract}
We introduce a novel input-idler-free non-degenerate phase sensitive amplifier (PSA) configuration and use it for simultaneous phase and amplitude regeneration of quadrature phase shift keyed (QPSK) signals demonstrated at symbol rates up to 56 Gbaud.

OCIS codes: (060.2320) Fiber optics amplifiers and oscillators, (190.4410) Nonlinear optics, parametric processes
\end{abstract}

\section{Introduction}

Advanced modulation formats such quadrature phase shift keying (QPSK) are increasingly viable for high capacity optical networks. In line with this, the development of techniques capable of processing multi-level signals represents the logical next step in the nascent field of all-optical information signal processing. Single bit-persymbol regeneration of phase encoded signals has been demonstrated using degenerate dual pump (2P) PSAs [1]; this approach has been significantly enhanced by a recent scheme allowing black-box operation, with the incorporation of an automatic pump-signal synchronization subsystem [2]. To regenerate QPSK signals, theoretical proposals have included the use of parallel BPSK/DPSK regenerators coherently combined at their outputs [3]. This parallelistic approach, often favoured in electronics, is complicated in optics due to the requirement to equalize and stabilise multiple optical paths (often $>100 \mathrm{~m}$ long with silica fiber implementations) in phase, polarization and propagation delay, which is both complicated and costly. Another proposal utilises a signal-idler conjugate pair transmitted down the link and combined in a non-degenerate (ND) PSA [4]; this however wastes valuable transmission bandwidth, and requires impractically low levels of residual dispersion post-compensation.

We recently introduced and demonstrated a new concept, enabling the first demonstration of all-optical QPSK phase regeneration [5]. This utilises a ND four wave mixing (FWM) based parametric amplifier, in which the phase modulated signal interacts with an idler wave bearing a multiple of the temporal phase modulation on the signal. For a QPSK signal, the idler field phase is required to be thrice the signal phase, which was achieved using a first stage incorporating a FWM based coherent phase multiplier, followed by a 2P ND PSA [5]. In this paper, we introduce a remarkable variant of that scheme, in which phase sensitive gain is obtained directly from a $2 \mathrm{P}$ ND parametric amplifier without an idler at the amplifier input, in apparent contradiction to the expected characteristics of a ND PSA. This device configuration, in addition to allowing QPSK phase regeneration in a simpler configuration relying on just one nonlinear element, provides significantly enhanced amplitude noise improvement. We utilise an equivalent time optical sampling oscilloscope to characterize the device's regenerative properties.

\section{Experimental Setup and Results}

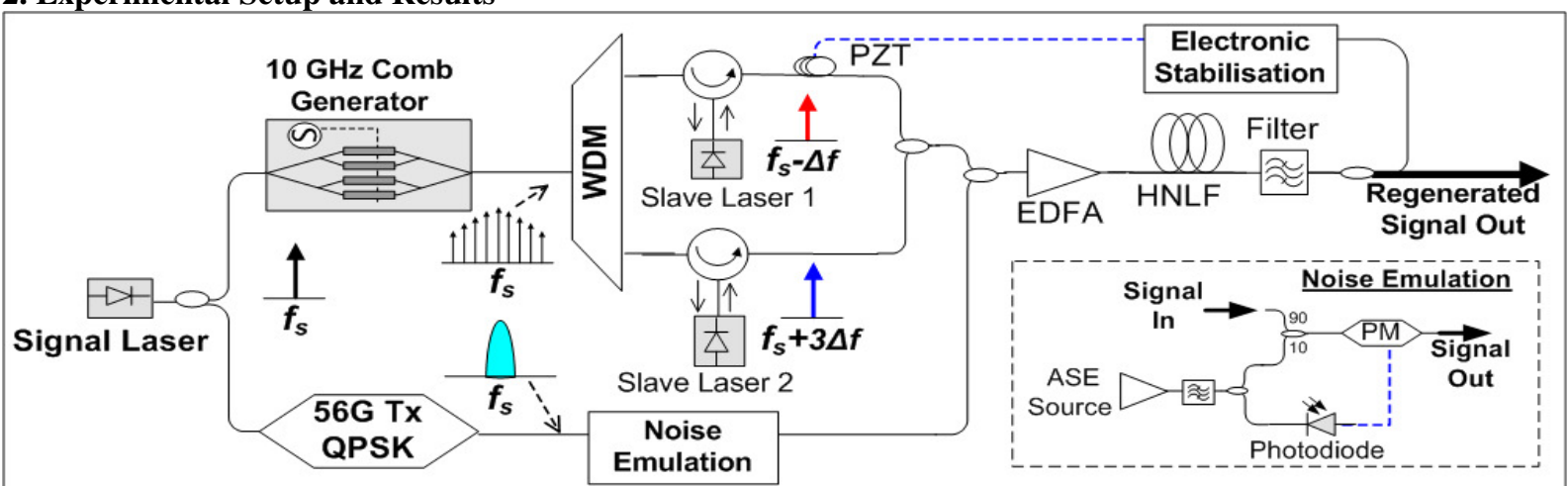

Figure 1: Regenerator setup, Tx - transmitter, WDM - wavelength division demultiplexer, PZT - piezo-electric fiber stretcher, EDFA - erbium doped fiber amplifier, PM - phase modulator, HNLF - highly nonlinear fiber, PM - phase modulator, ASE -amplified spontaneous emission. HNLF parameters are length $300 \mathrm{~m}$, nonlinear coefficient $11.6 / \mathrm{W} / \mathrm{km}$, zero dispersion wavelength $1553 \mathrm{~nm}$ and $1550 \mathrm{~nm}$ slope $0.018 \mathrm{ps} / \mathrm{nm} 2 / \mathrm{km}$. 


\section{OMT4.pdf}

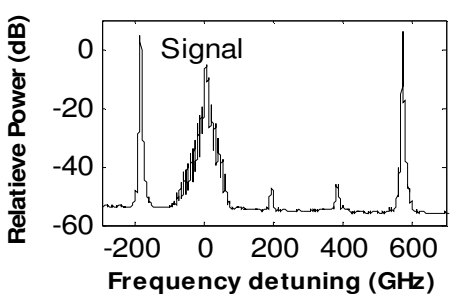

Figure 2: PSA input spectrum, signal located at $0 \mathrm{GHz}$ detuning.

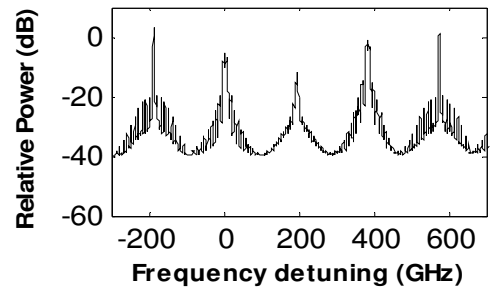

Figure 3: PSA output spectrum, signal located at $0 \mathrm{GHz}$ detuning, output idler at $+380 \mathrm{GHz}$

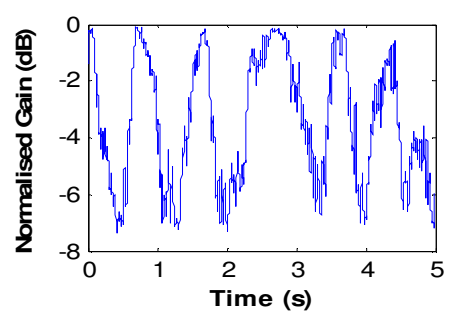

Figure 4: Normalised PSA gain fluctuations with electronic stabilisation turned off.

The output of a CW laser at $1555.7 \mathrm{~nm}$ was split into two, with one portion coupled into a $10 \mathrm{GHz}$ comb generator (see Fig. 1). Comb lines at -190 and $+570 \mathrm{GHz}$ detuning were injected into semiconductor lasers, providing two pump beams phase locked to the signal carrier. For black-box implementations, the pump-signal synchronization scheme demonstrated in [5] would replace this linear comb. The rest of the signal light was modulated with a pseudo-random binary sequence to generate single polarization QPSK. This was sent through a noise additive module to emulate the effects of linear (related to quantum noise and ASE) and nonlinear phase noise (related to nonlinear amplitude to phase conversion). This module (shown as an inset in Fig. 1) comprised an ASE source whose output was split into two, one portion being detected and the resulting electrical white noise being used to drive a LiNbO3 phase modulator through which the signal was passed, and the other portion being optically combined with the signal in a coupler. The signal was then combined with the pumps and all the waves were amplified in an EDFA, leading to $50 \mathrm{~mW}$ of signal power and $250 \mathrm{~mW}$ power per pump. They were then sent into an HNLF (OFS, Denmark). This had a strain gradient to increase its stimulated Brillouin scattering (SBS) threshold, allowing the use of continuous wave pumps. Slow thermo-acoustic relative phase drifts were suppressed by monitoring the signal power at the PSA output and controlling the PZT. The signal was then assessed using an EXFO constellation analyzer (PSO-200) based on all-optical sampling capable of operation up to 100 Gbaud.

Table 1: Regenerator performance. A - Input phase noise only; B- Phase and amplitude noise; C- No noise;

D- Phase noise only. $\sigma_{\Delta \theta}^{2}$ is the phase error variance, $\sigma_{\Delta r h o}^{2}$ is the normalised amplitude noise variance;

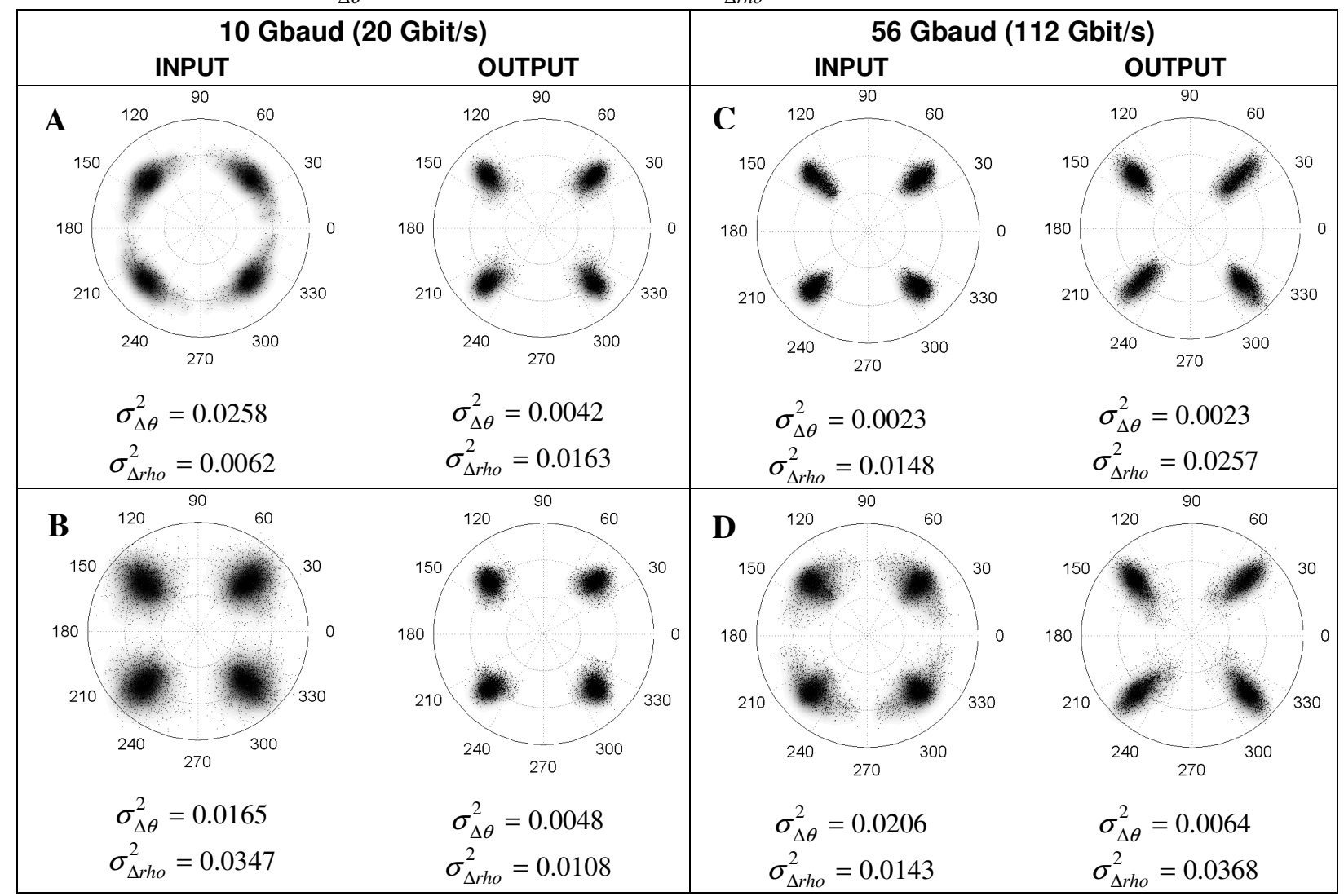


The input and output spectra to the PSA are shown in Fig. 2 and Fig. 3 respectively. At the input to the PSA, there is negligible power at the idler frequency $(+380 \mathrm{GHz}$ detuning); a very weak component emanating from weak FWM in the high power EDFA is visible but at $-40 \mathrm{~dB}$ relative to the signal this does not affect the subsequent parametric interaction as verified by numerical simulations. $7 \mathrm{~dB}$ phase sensitive gain variation was obtained at the PSA output as measured with the feedback to PZT turned off (Fig. 4). The spectrum at the output to the PSA (Fig. 3) suggests two separate interactions occur simultaneously - first, the presence of the strong component at $+190 \mathrm{GHz}$ detuning indicates coherent phase multiplication via mixing of the signal with the pump at $-190 \mathrm{GHz}$, and the strong idler at $+380 \mathrm{GHz}$ indicates conventional 2P phase insensitive amplification. We believe the coherent interplay between these two interactions leads to the phase sensitive gain, with the transfer characteristics originating from the same principles as in [5]. This will be analytically and numerically studied further in due course.

To characterise the regenerator, colorgrade signal constellations including data on the phase error variance and normalised variance of amplitude noise are shown in Table 1. While this statistical information would be more robust if obtained from a homodyne receiver without digital phase compensation for the intradyne local oscillator, it is still useful for quantifying relative signal improvements derived from the regenerator. The regenerator was first assessed at 10 Gbaud with added phase noise only, emulating the nonlinear regime. Pseudo-Gaussian phase fluctuations would be expected at the input, with an artificial roll-off at the tails of the distribution due to saturation of the photodiode for high ASE levels. The regenerator desirably reduced the phase error variance by a factor of 6 , while the amplitude noise variance only increased by 2.6 (Table 1, Cell A), suggesting an overall benefit as the input BER is dominated by the phase noise. This phase noise reduction is comparable to the numerically predicted factor of 5.5 for the parallel BPSK regenerator scheme [3], denoting that the inline compactness of our approach does not come with an associated performance penalty. For QPSK, a comparison with studies on the impact of phase estimation errors on BER suggests that the output phase error variance of $0.0042 \operatorname{rad}^{2}$ (Table 1, Cell A) approximately corresponds to an SNR penalty under $0.5 \mathrm{~dB}$ for a BER of $10^{-4}$, while the input variance corresponds to a penalty $>>4 \mathrm{~dB}[6]$. This nonlinear phase noise reduction implies that i) the reach of the transmission span can be increased ii) the tolerance to nonlinearity is significantly enhanced; hence higher receiver OSNRs can be envisaged. In the presence of linear noise emulated by ASE loading (hence degraded OSNR with both phase and amplitude fluctuations), the phase error variance and normalised amplitude variance are simultaneously reduced by approximately 3.2, indicating even better net regenerator performance (Table 1, Cell B). This ability to concurrently reduce both absolute phase and amplitude variance prior to the receiver suggests that the regenerator should provide significant signal quality improvement if deployed before a differential receiver, which normally requires a trade off between reduced complexity and lower noise tolerance compared to a fully coherent one.

The symbol rate was increased to 56 Gbaud. Without added noise, the regenerator preserves the phase quality at the expense of some amplitude noise (Table 1, Cell C). We believe this is a result of multiple parametric interactions occurring in the PSA that transfer some amplitude noise to the signal. In the presence of phase noise, the phase error variance is reduced by a factor of 3.2, with increased amplitude noise at the output (Table 1, Cell D). For QPSK however, in which the information is solely contained in the phase, this increased nonlinear phase noise tolerance would translate to increased reach or higher signal launch powers to improve OSNR at the receiver.

\section{Conclusions}

We have introduced and demonstrated a novel degenerate phase sensitive amplifier configuration, notable for the absence of an idler field at the device input. This has been used to demonstrate QPSK phase and amplitude regeneration at 56 Gbaud, providing signal improvements in emulated linear and nonlinear phase noise regimes.

\section{Acknowledgement}

We thank OFS Denmark and Eblana Photonics for the provision of strained HNLF semiconductor laser respectively and especially acknowledge the support of L. Grüner-Nielsen (OFS) and R. Phelan (Eblana Photonics). This research has received funding from the European Communities Seventh Framework Programme FP/2007-2013 under grant agreements 224547 (PHASORS) and 216863 (BONE).

\section{References}

[1]K. Croussore, I. Kim, Y. Han, C. Kim, G. Li, and S. Radic, "Demonstration of phase-regeneration of DPSK signals based on phase-sensitive amplification," Opt. Express, 13, 3945-3950 (2005).

[2]R. Slavik, et. al.,"All-optical phase and amplitude regenerator for next-generation telecommunications systems," Nat Photon 4, 690-695 (2010) [3]Z. Zheng, L. An, Z. Li, X. Zhao, and X. Liu, "All-optical regeneration of DQPSK/QPSK signals based on phase-sensitive amplification," Optics Communications 281, 2755-2759 (2008).

[4] M. Vasilyev, "Distributed phase-sensitive amplification," Opt. Express 13, 7563-7571 (2005).

[5] J.Kakande, A.Bogris, R.Slavik, F.Pamigiani, D.Syvridis, P.Petropoulos, and D.J.Richardson, "First demonstration of all-optical QPSK signal regeneration in a novel multi-format phase sensitive amplifier", in Proc. of ECOC 2010, Turin (Italy), Sept. 2010, post deadline PD 3.3

[6] C. Yu, S. Zhang, P. Kam, and J. Chen, "Bit-Error Rate Performance of Coherent Optical M-ary PSK/QAM using Decision-Aided Maximum Likelihood Phase Estimation," Opt. Express 18, 12088-12103 (2010). 